\title{
Characterization of Optimal Strain, Frequency and Duration of Mechanical Loading on Skeletal Myotubes' Biological Responses
}

\author{
ATHANASIOS MOUSTOGIANNIS*, ANASTASSIOS PHILIPPOU*, EVANGELOS ZEVOLIS*, \\ ORJONA TASO, ANTONIOS CHATZIGEORGIOU and MICHAEL KOUTSILIERIS \\ Department of Physiology, Medical School, National and Kapodistrian University of Athens, Athens, Greece
}

\begin{abstract}
Background/Aim: Mechanical loading of differentiated myoblasts in vitro may mimic loading patterns of skeletal muscle in vivo. However, it is still uncharacterized the loading conditions that can produce the most effective muscle cells' biological responses, in vitro. This study investigated the effects of different loading protocols on the expression of myogenic regulatory factors, anabolic, atrophy and pro-apoptotic factors in skeletal myotubes. Materials and Methods: C2C12 myoblasts were differentiated and underwent various stretching protocols by altering their elongation, frequency and duration, utilizing an in vitro cell tension system. The loading-induced expression changes of MyoD, Myogenin, MRF4, IGF-1 isoforms, Murf1, Atrogin, Myostatin, Foxo and Fuca were measured by Real TimePCR. Results: Stretching by 2\% elongation at $0.25 \mathrm{~Hz}$ for 12 $h$ was overall the most effective in inducing beneficial responses. Conclusion: A low strain, low frequency intermediate duration stretching can most effectively upregulate myogenic/anabolic factors and down-regulate proapoptotic and atrophy genes in myotubes.
\end{abstract}

Skeletal muscle tissue has the ability to adapt to mechanical stimuli by changing its mass and overall contractile phenotype via the activation of mechanotransduction and intracellular signaling mechanisms $(1,2)$. Mechanosensitive complexes (mechanosensors) situated in the muscle cell membrane are able to sense alterations and appear to have an important role

This article is freely accessible online.

*These Authors contributed equally to this study.

Correspondence to: Dr. Michael Koutsilieris, MD, Ph.D., Department of Physiology, Medical School, National \& Kapodistrian University of Athens, 75 Micras Asias, Goudi-Athens, 115 27, Greece. Tel: +30 2107462507, Fax: +30 2107462571, e-mail: mkoutsil@med.uoa.gr

Key Words: Mechanical loading, mechanotransduction, myogenesis, myotubes. in promoting muscle cell growth, metabolism and survival (3). Indeed, cellular mechanotransduction can induce and modulate a wide range of biological responses, such as gene expression, protein synthesis and secretion, cell proliferation, survival and apoptosis $(3,4)$. Moreover, mechanical signals that regulate muscle growth and regeneration, in both physiological and pathological conditions, can also affect the differentiation of myoblasts to mature myotubes during myogenesis (5).

Myogenic differentiation is driven by multiple signal transduction pathways which coordinate the balance between muscle growth and atrophy, or protein synthesis and protein degradation $(6,7)$. Specifically, the myogenic program is regulated by four, structurally similar transcriptional activators, the Myogenic Regulatory Factors (MRFs) Myf5, MyoD, Myogenin and MRF4 $(8,9)$. In addition, Insulin-like growth factor-1 (IGF-1) signaling has been implicated in the process of myogenesis $(10,11)$ as it is a key factor in the regulation of skeletal muscle development and growth (12-14). Its upregulation leads to muscle hypertrophy (15) while, interestingly, potentially differential actions of IGF-1 isoforms in skeletal muscle growth and regeneration have been proposed (16-19).

On the other hand, muscle-specific atrophy factors along with pro-apoptotic factors are negative regulators of muscle growth and development (20). In particular, muscle protein degradation occurs by the activation of muscle-specific ubiquitin ligases, Atrogin-1 (MaFbx) and muscle ringer finger1 (MuRF1), along with the down-regulation of pro-growth genes (21-23). Moreover, Atrogin-1 promotes MyoD degradation and deregulates transcription factors and mitochondrial enzymes $(24,25)$. Similarly, MuRF1 has been reported to interact with and control the half-life of many important muscle structural proteins (26). In addition, increased myostatin activity prevents muscle growth, inhibits myoblast differentiation and protein synthesis, reduces myotube size and inhibits IGF-1-induced myotube hypertrophy (27-29). Furthermore, it has been reported that when skeletal muscle cells undergo atrophy, a subset of myoblasts exhibits apoptosis (30) and important pro-apoptotic factors, such as FoxO and p53, have been negatively implicated in muscle cell growth and differentiation. In particular, overexpression of FoxO has been 
found to dramatically inhibit $\mathrm{C} 2 \mathrm{C} 12$ myoblasts differentiation (31). Also, p53 and its downstream effectors are required for an inflammatory cytokine-mediated inhibition of myogenic differentiation in vitro (32).

The expression of muscle growth/atrophy-associated factors such as the afore-mentioned has been implicated in the unloading-induced atrophy mechanisms and found to be influenced by the lack of external load of skeletal muscle in vivo (33). Thus, understanding the contribution of the particular inputs of such factors on muscle cell adaptation to mechanical loading has been challenging. However, defining the molecular responses of muscle cells to mechanical stimuli in vivo is technically very difficult $(34,35)$, because skeletal muscle contains several cell types that influence its responses to loading. Given the complexity of the in vivo myogenic adaptation in response to exercise-induced external loading, in vitro models of mechanical loading applied on muscle cells are crucial for understanding the cellular and molecular mechanisms that mediate loading-induced adaptations. The myogenic differentiation of myoblast cell lines into myotubes represents a well-established model of skeletal muscle differentiation and in the last decade in vitro models have been adopted to particularly mimic in vivo muscle loading conditions $(1,36)$.

The aim of this study was to characterize the efficacy and efficiency of various in vitro mechanical loading protocols on gene expression responses of differentiated myoblasts, associated with anabolic, atrophy and pro-apoptotic factors involved in their myogenic lineage. We set the hypothesis that expression responses elicited would differ depending on the loading characteristics of the protocols used, thus indicating loading-specific, detrimental or beneficial effects on myotubes in vitro.

\section{Materials and Methods}

C2C12 cell culture. The $\mathrm{C} 2 \mathrm{C} 12$ cell line of mouse myoblasts was obtained from American Type Culture Collection (Manassas, VA, USA) and cultured as previously described (37). Briefly, cells were grown in Dubecco's modified Eagle's medium (DMEM) supplemented with $10 \%$ fetal bovine serum (FBS), plus $1 \%$ penicillin/streptomycin at $37^{\circ} \mathrm{C}$ in a humidified atmosphere of $5 \%$ $\mathrm{CO}_{2}$ in air, while medium was changed every other day. The $\mathrm{C} 2 \mathrm{C} 12$ myoblasts were seeded onto 6-well flexible-bottomed culture plates coated with Collagen I (Flex I Culture Plates Collagen I; Flexcell International, Hillborough, NC, USA) and maintained in growth media until $70 \%$ to $80 \%$ confluent, then switched to differentiation media (2\% horse serum, $1 \%$ of penicillin/streptomycin in DMEM). Myoblasts were allowed to differentiate into multinucleated myotubes for a 10-day period during which media was changed every other day before stretching as described below.

Myotube mechanical loading. Differentiated myotubes were stretched using the Flexcell FX-4000 strain unit (Flexcell International) that produces isotropic two-dimensional (biaxial) strain of cells cultured on the flexible surface (silicone membrane) of the culture plates, again at $37^{\circ} \mathrm{C}$ in a humidified atmosphere of $5 \% \quad \mathrm{CO}_{2}$. Briefly, myotubes were subjected to five different stretching protocols: a) $15 \%$ elongation (strain) at a frequency of $1 \mathrm{~Hz}$ for $15 \mathrm{~min}, \mathrm{~b}) 10 \%$ strain at $1 \mathrm{~Hz}$ for $1 \mathrm{~h}, \mathrm{c}) 10 \%$ elongation at $0.25 \mathrm{~Hz}$ for $1 \mathrm{~h}, \mathrm{~d}) 2 \%$ strain at $0.25 \mathrm{~Hz}$ for $12 \mathrm{~h}$, or e) $2 \%$ strain at $0.25 \mathrm{~Hz}$ for $24 \mathrm{~h}$.

Cell lysis and RNA extraction. Cell extracts were obtained by cell lysis using NucleoZOL (Mecherey-Nagel, Duren, Germany) $12 \mathrm{~h}$ after the completion of the stretching protocol, while control (non-stretched) myotubes were also harvested $12 \mathrm{~h}$ after the end of each stretching protocol. Total RNA was isolated from the lysates according to the manufacturer's recommendations. The extracted RNA was dissolved in RNAases free water (Invitrogen, Carlsbad, CA, USA) and the concentration and purity were determined spectrophotometrically (Thermo Nanodrop 2000, Thermo Scientific ${ }^{\mathrm{TM}}$, Waltham, MA, USA) by absorption at 260 and $280 \mathrm{~nm}$. The integrity of total RNA was confirmed by visual inspection of the electrophoretic pattern of $18 \mathrm{~S}$ and 28S ribosomal RNA in ethidium bromide-stained $1 \%$ agarose gels under ultraviolet (UV) light. The total RNA samples were stored at $80^{\circ} \mathrm{C}$ until further analyses for the determination of the mRNA levels of the genes of interest by reverse transcription and semi-quantitative real-time PCR procedures.

Reverse transcription and real-time PCR. Total RNA from each sample was used to produce single-stranded cDNA by reverse transcription using reverse transcriptase ProtoScript II (NEB, Ipswich, MA, USA) and the resultant cDNAs were utilized in realtime PCR. More specifically, for the reverse transcription $1 \mu \mathrm{g}$ of total RNA from each sample was mixed with random primers mix (300 ng/reaction), oligod(T) $23 \mathrm{VN}$ (300 ng/reaction) and nucleasefree water in a total volume of $8 \mu \mathrm{l}$, heated at $65^{\circ} \mathrm{C}$ for $5 \mathrm{~min}$ and then placed on ice. Next, the samples were mixed with $10 \mu \mathrm{l}$ ProtoScript II Reaction Mix and $2 \mu$ Protoscript II Enzyme mix and incubated consecutively at $25^{\circ} \mathrm{C}$ for $5 \mathrm{~min}$ and at $45^{\circ} \mathrm{C}$ for $1 \mathrm{~h}$ according to manufacturer's recommendations. At the final step of the reverse transcription, the samples were heated at $80^{\circ} \mathrm{C}$ for $5 \mathrm{~min}$, to inactivate the enzyme, and stored at $-20^{\circ} \mathrm{C}$.

Real-time PCR analyses were performed using the Bio-Rad 96well iCycler thermal cycler (Bio-Rad iQ5 Real-Time PCR Detection System, Hercules, CA, USA) and Bio-Rad reagents ( $\mathrm{iQ}^{\mathrm{TM}}$ SYBR Green Supermix). The primer set sequences used for the specific detection of IGF-1 isoforms (IGF-1Ea, IGF-1Eb), MRFs (MyoD, Myogenin, Mrf4), atrophy (Myostatin, MuRF1, Atrogin-1) and proapoptotic factors (FoxO, FUCA, p53), are shown in Table I. To prevent detection of genomic DNA, the primer sets were designed to lie within different exons while, particularly, each set of primers for the detection of the IGF-1 isoforms was specific to detect only one specific IGF-1 transcript. Each PCR reaction contained $50 \mathrm{ng}$ of cDNA, $12.5 \mu \mathrm{l}$ SYBR green master mix, $0.4 \mu \mathrm{M}$ of each primer, and nuclease free water to a total volume of $20 \mu$ l. The real-time PCR parameters were the following: initial denaturation at $95^{\circ} \mathrm{C}$ for $5 \mathrm{~min}$ followed by 40 cycles of $30 \mathrm{~s}$ at $95^{\circ} \mathrm{C}, 30 \mathrm{~s}$ at $62^{\circ} \mathrm{C}$ for annealing, and $30 \mathrm{~s}$ at $72^{\circ} \mathrm{C}$ for extension. Transcript levels of the genes of interest were assessed by automatically calculating the threshold cycle $(\mathrm{Ct})$ as the number of cycles at which the measured fluorescence exceeds the threshold for detection. To normalize the amount of total RNA present in each PCR reaction and the mRNA expression (relative quantification-dCt) of the genes of interest, 
Table I. The sequence of the specific sets of primers used for RT-PCR analyses.

\begin{tabular}{lll}
\hline Target gene & $5^{\prime}$ '-3' (forward) primer sequence & 3'-5' (reverse) primer sequence \\
\hline GAPDH & CAA CTC CCT CAA GAT TGT CAG CAA & GGC ATG GAC TGT GGT CAT GA \\
MyoD & TGC TCC TTT GAG ACA GCA GA & AGT AGG GAA GTG TGC GTG CT \\
Myogenin & AGG AGA GAA AGA TGG AGT CCA GAG & TAA CAA AAG AAG TCA CCC CAA GAG \\
MRF 4 AGG GCT CTC CTT TGT ATC CAG & TGG AAG AAA GGC GCT GAA GA \\
$I G F-1 E b$ & GTG GAC GCT CTT CAG TTC GT & GCT TCC TTT TCT TGT GTG TCG ATA G \\
$F U C A$ & GTC CCC AGC ACA CAT CGC G & TCT TTT GTG CAA AAT AAG GCG TA \\
FoxO & TTT GGT CGG TGA GTT GGG AG & CCA TTC CAA GAG CGA GTG GT \\
TP53 & AGT GGA TGG TGA AGA GCG TG & GAA GGG ACA GAT TGT GGC GA \\
Myostatin & GAG AGA CCG CCG TAC AGA AG & AGC AGT TTG GGC TTT CCT CC \\
Atrogin-1/MAFbx & CTG TAA CCT TCC CAG GAC CA & GCA GTC AAG CCC AAA GTC TC \\
\hline
\end{tabular}

glyceraldehyde 3-phosphate dehydrogenase (GAPDH) was used as housekeeping gene (internal standard). Each sample was analyzed in duplicate, and the resulting data were averaged. A melting curve (Tm) was also generated by the Bio-Rad iQ5 Real-Time PCR Detection System software after the final cycle for each experimental sample, by continuous monitoring the Bio-Rad SYBR fluorescence throughout the temperature ramp from $70^{\circ} \mathrm{C}$ to $95^{\circ} \mathrm{C}$. The specificity of the primers for the corresponding transcript was also confirmed by the melting curve analysis of samples, where there was only one melting curve for each sample and electrophoretic analysis of the real-time PCR products further verified the specificity of the transcript of each gene of interest. Control for specificity included cDNA-free reactions and template-free reactions.

Statistical analysis. One-way analysis of variance (ANOVA) with Dunn's Multiple Comparison post-hoc test was used for statistics, using GraphPad Prism 5. All experiments were performed in triplicate and data are presented as mean \pm standard error of the mean (S.E.M). The level of statistical significance was set at $p<0.05$.

\section{Results}

Myogenic regulatory factors. In order to investigate the potential effects of mechanical loading on the myogenic lineage of differentiated myoblasts, we examined the expression levels of both early (MyoD) and late (Myogenin, MRF4) differentiation factors in skeletal myotubes. It was found that only the low frequency $(0.25 \mathrm{~Hz})$, low elongation (2\%) of longer duration stretching protocols induced significant changes in the expression levels of those MRFs compared to both the control (no stretch) and the higher elongation or frequency conditions (Figure 1A-C). Interestingly, MyoD exhibited differential responses to mechanical loading compared to the late differentiation MRFs; only the $24 \mathrm{~h}$ stretching induced a significant upregulation of MyoD compared to control, in contrast with Myogenin and MRF4 expression, whose expressions were higher in the $12 \mathrm{~h}$ stretching condition compared to both the control and the $24 \mathrm{~h}$ stretching (Figure 1A-C). Moreover, the 12-h loading protocol was the only that induced significant changes in the expression of MRF4 compared to any other (control or stretching) condition (Figure 1C).

Anabolic factors. As IGF-1 is a major regulator of skeletal muscle development and growth that can induce hypertrophy and block atrophy, we examined the effects of different stretching protocols on the expression of IGF-1 isoforms in skeletal myotubes. For the IGF-1Ea, the most effective protocol was that of $2 \%$ strain at $0.25 \mathrm{~Hz}$ for $24 \mathrm{~h}$, increasing its expression compared to both the control and the other stretching protocols used (Figure 2A). Interestingly, a differential response was revealed regarding the IGF-1Eb isoform, for which the low frequency protocol $(0.25 \mathrm{~Hz})$, regardless of its elongation or duration, resulted in the upregulation of this isoform (Figure $2 \mathrm{~B}$ ).

Atrophy factors. In parallel with the effects of mechanical loading on the anabolic factor IGF-1, we also examined the effects of different stretching protocols on the expression of muscle atrophy genes. It is noteworthy that in contrast to the IGF-1 isoforms responses, the stretching protocol with the highest elongation (15\%) and frequency $(1 \mathrm{~Hz})$ along with the shortest duration $(15 \mathrm{~min})$ resulted in significant increases of the atrophy genes expression, particularly of myostatin and Atrogin-1, compared to controls (Figure 3A and $\mathrm{C}$ ). On the contrary, the low elongation (2\%), low frequency $(0.25 \mathrm{~Hz})$ for $12 \mathrm{~h}$ stretching was the only one to cause a significant decrease in the expression of all three atrophy genes examined (Figure 3A-C). Moreover, the same, 12-hrs low elongation and low frequency protocol resulted in the more pronounced down-regulation of myostatin and Atrogin-1 compared to the high elongation/high frequency protocols (Figure 3A and C). 

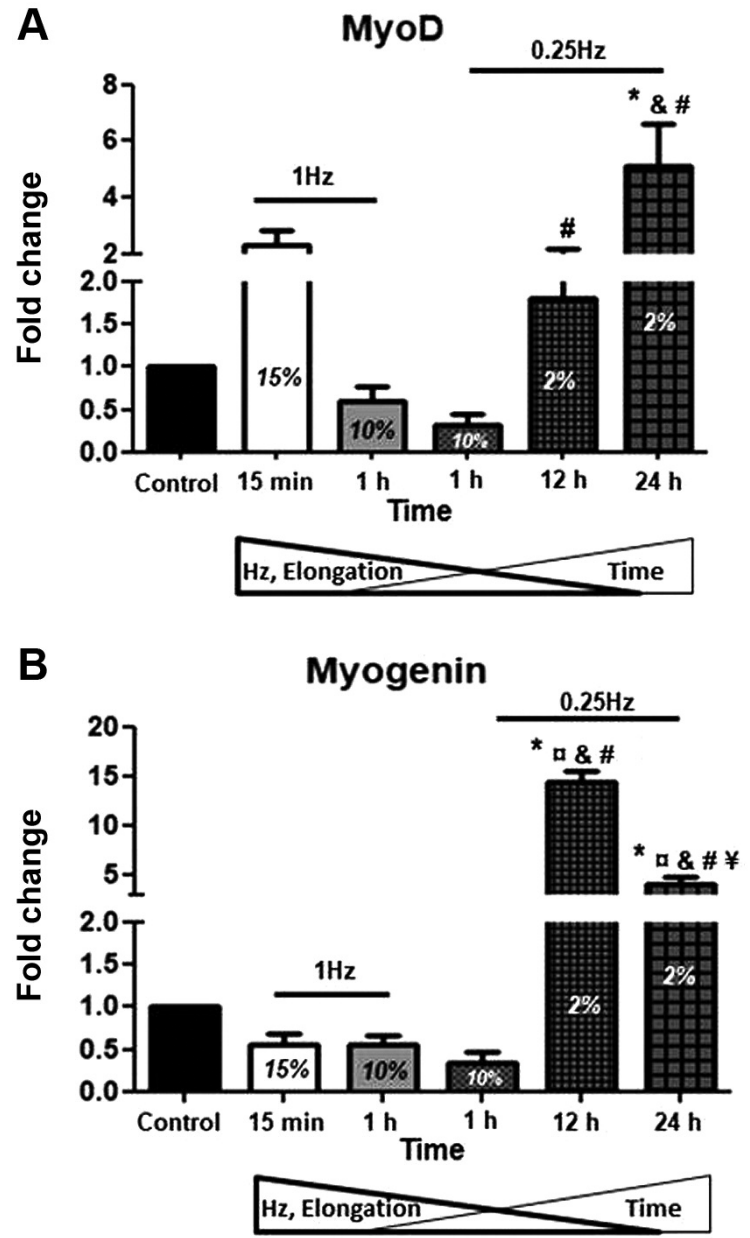

Pro-apoptotic factors. Along with the muscle atrophy genes, we further examined the loading-induced regulation of apoptosis-related factors in the skeletal myotubes. Our results showed that the stretching protocol of $10 \%$ elongation at either low $(0.25 \mathrm{~Hz})$ or high $(1 \mathrm{~Hz})$ frequency for $1 \mathrm{~h}$ led to significant up-regulation of the pro-apoptotic factors compared to controls as well as to the $15 \%$ elongation for 15 min stretching (Figure 4A-C), indicating that the duration of stretching may determine the particular differences between the high-strain protocols. On the contrary, and similarly to the atrophy genes responses, significant decrease in the expression of those three pro-apoptotic factors was observed in the low elongation $(2 \%)$, low frequency $(0.25 \mathrm{~Hz})$ for 12 $\mathrm{h}$ stretching protocol compared to control condition (Figure 4A-C). Moreover, the low elongation, low frequency of the longer durations (12 and $24 \mathrm{~h}$ ) protocols resulted in more pronounced down-regulation of those factors compared to the high elongation $(10 \%)$ /short duration $(1 \mathrm{~h})$ protocols (Figure 4A-C).

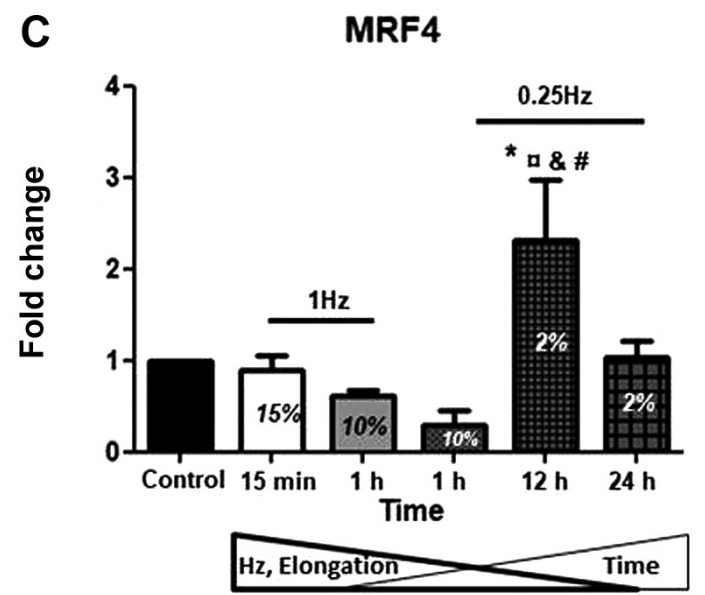

Figure 1. Effects of cyclic mechanical stretch on the expression of MRFs. Quantitative analysis of (A) MyoD, (B) Myogenin and (C) MRF4 mRNA expression in myotubes subjected to mechanical stretch compared to control (non-stretched myotubes); The mRNA values of MRFs in stretched myotubes were normalized to the corresponding GAPDH $m R N A$ and are expressed as fold changes compared to control. Percentages on the columns represent the degree of elongation of the cell culture surface. Note that in the selected stretching protocols as the elongation and frequency $(\mathrm{Hz})$ decrease the duration (Time) increases. * significantly different compared to control; " significantly different compared to $15 \%$ elongation; \& significantly different compared to $10 \%$ elongation/1 Hz; \#significantly different compared to $10 \%$ elongation $/ 0.25 \mathrm{~Hz}$; $¥$ significantly different compared to $2 \%$ elongation/12 h; Mean+SE of 3 independent experiments performed in triplicate; $p<0.05$.

\section{Discussion}

This study examined the effects of various in vitro cell stretching protocols on gene expression responses associated with the myogenic lineage of differentiated $\mathrm{C} 2 \mathrm{C} 12$ myotubes, in order to reveal potential loading-specific, detrimental or beneficial effects on the myogenic program in myotubes, depending on the loading characteristics of the protocols used. The expression of myogenic, anabolic, atrophy and pro-apoptotic factors were measured $12 \mathrm{~h}$ after the completion of each stretching protocol to determine durable, persistently triggered rather than short transcriptional responses. Our main findings demonstrated that a low strain, low frequency for an intermediate duration mechanical stretching was overall the most effective in inducing beneficial responses in skeletal myotubes, by upregulating MRFs and IGF-1 while down-regulating atrophy and pro-apoptotic factors. Furthermore, the present study revealed that the late myogenic determination factors, 
Myogenin and MRF4, exhibited differential responses to mechanical loading compared to the early differentiation factor MyoD. Similarly, a differential response of IGF-1Eb isoform to the cell stretching protocols was revealed compared with the other IGF-1 isoform, IGF-1Ea. Interestingly and in contrast to the responses of the myogenic and anabolic factors, the stretching protocol with the highest strain and frequency along with the shortest duration resulted in increased expression of the atrophy genes. Moreover, the low strain, low frequency and longer duration (12 and $24 \mathrm{~h}$ ) stretching protocols resulted in the more pronounced downregulation of the pro-apoptotic factors compared to the high strain, short duration protocols.

The ability of mechanosensitive cells to sense external mechanical stimuli and convert them into electrochemical and biochemical signals (mechanotransduction) is critical for the maintenance of cellular and tissue homeostasis (38-40). In particular, mechanical loading can cause changes in patterns of gene expression in skeletal muscle, influencing protein synthesis and stability and affecting muscle metabolism, while there is a growing body of evidence showing that mechanical cues modulate many aspects of myocyte function, including proliferation and myogenic differentiation (10). The myogenic differentiation program is regulated by MRFs, a family of muscle-specific transcription factors considered myogenic determinants, which share the ability to convert various differentiated cell types to myogenic and direct progenitor cells to establish the skeletal muscle lineage (41). In particular, Myogenin, lying genetically downstream, along with MyoD and MRF4, activate the myogenic differentiation program $(8,42)$. MyoD promotes myoblasts progression to terminal differentiation (43) while it seems, along with Myogenin, to directly or indirectly activate the expression of MRF4 in the later stages of myogenic differentiation (44). Myogenin, as MRF4, is a late differentiation factor stimulating terminal myogenic differentiation and its expression can be induced by IGF-1 (45).

This study showed that only the low frequency/low strain of longer duration stretching conditions induced significant changes in the expression of those MRFs (Figure 1A-C) while, particularly, only the longest duration ( $24 \mathrm{~h}$ ) stretching induced a significant up-regulation of MyoD, versus Myogenin and MRF4, whose expressions were higher in the $12 \mathrm{~h}$ stretching condition. These findings are similar with those reported by other studies that examined the responses of MyoD after cyclic tensile strain for 24,48 or $72 \mathrm{~h}$ in differentiated myoblasts, or regarding Myogenin and MRF4 responses to cyclic mechanical strain (46-48). Our findings suggest that those MRFs, at least at this (later) stage of myogenic differentiation, are responsive to mechanical loading and more sensitive particularly to long-term, low elongation stretching. Moreover, the time-dependent differential responses of MyoD compared with Myogenin
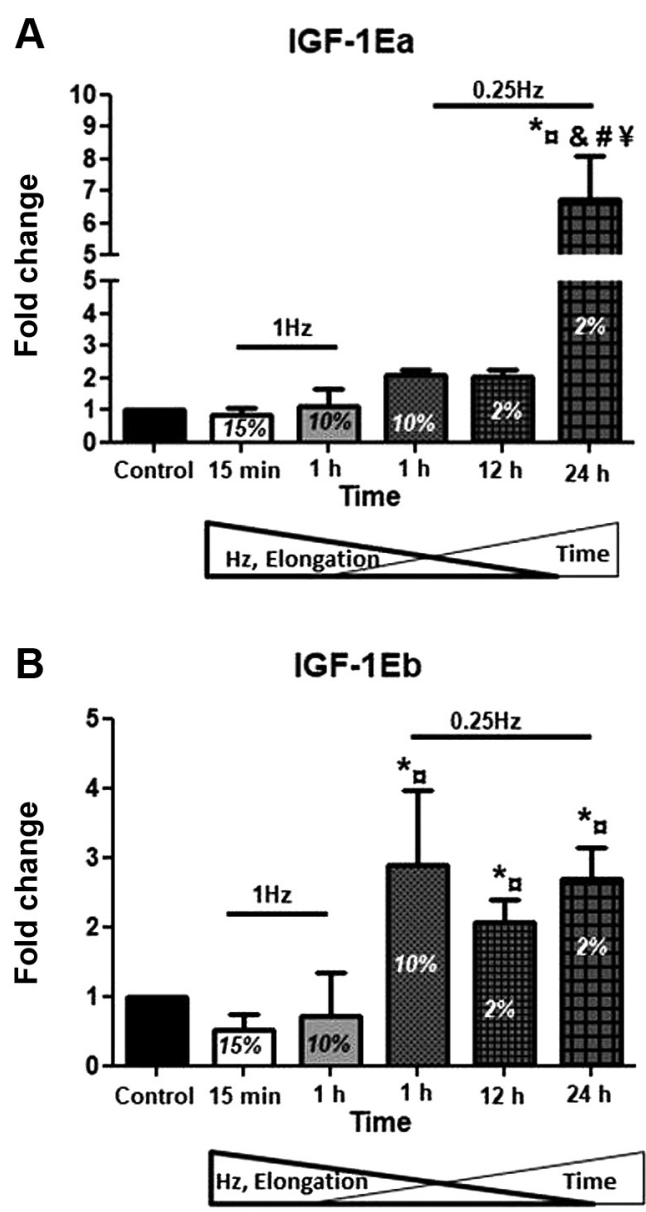

Figure 2. Effects of cyclic mechanical stretch on the expression of IGF1 isoforms. Quantitative analysis of (A) IGF-1Ea and (B) IGF-1Eb mRNA expression in myotubes subjected to mechanical stretch compared to control (non-stretched myotubes); The mRNA values of IGF-1 isoforms in stretched myotubes were normalized to the corresponding GAPDH $m R N A$ and are expressed as fold changes compared to control. Percentages on the columns represent the degree of elongation of the cell culture surface. Note that in the selected stretching protocols as the elongation and frequency $(\mathrm{Hz})$ decrease the duration (Time) increases. *significantly different compared to control; ${ }^{a}$ significantly different compared to $15 \%$ elongation; \& significantly different compared to $10 \%$ elongation/1 Hz; " significantly different compared to $10 \%$ elongation $/ 0.25 \mathrm{~Hz}$; ${ }^{¥}$ significantly different compared to $2 \%$ elongation $/ 12 \mathrm{~h}$.

and MRF4 to stretching may reflect a different responsiveness of the early vs late myogenic factors to mechanical stimuli, possibly depending on their distinct role(s) in that stage of the myogenic differentiation program.

Mechanical stretching of skeletal muscle cells both in vivo and in vitro can lead to the up-regulation of many growth factors, such as IGF-1, eventually leading to the enhancement of protein synthesis and muscle cell growth 


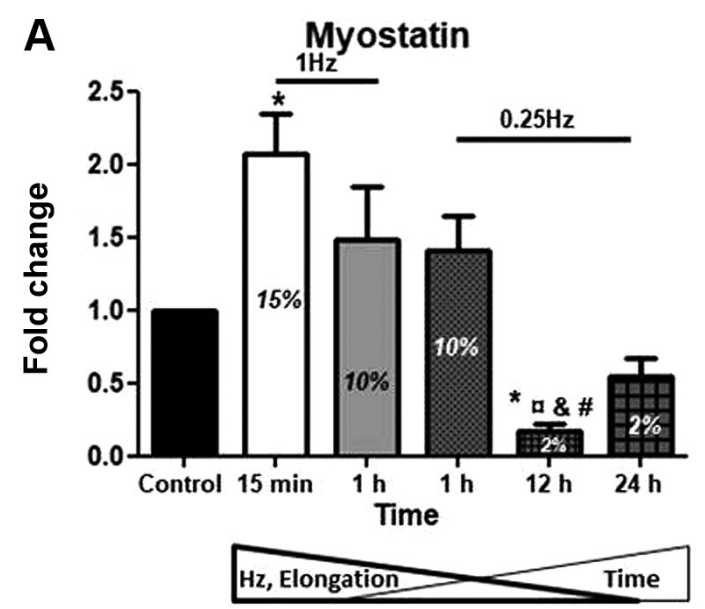

B

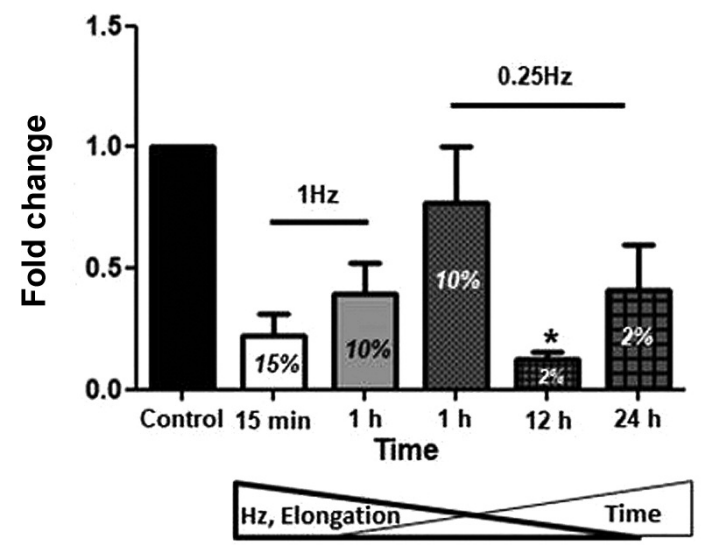

(24-26). Indeed, IGF-1 is a major anabolic factor involved in myogenesis and muscle hypertrophy $(11,49-51)$ and its up-regulation in response to mechanical loading observed in this study is in line with findings of previous studies (51-53). However, to the authors' best knowledge, this is the first study investigating the distinct expression profiles of IGF-1 isoforms following mechanical loading of myotubes, in vitro. Our data specifically showed that both isoforms appear to be up-regulated by low frequency stretching protocols, while differential responses were also revealed; IGF-1Ea found to be responsive only to the longest duration ( $24 \mathrm{~h})$ of stretching, while IGF-1Eb exhibited responsiveness to all low-frequency protocols used, regardless of their duration. These findings suggest that IGF-1 isoforms need low frequency loading to be activated, while their distinct, timedependent responses to stretch may reflect different biological roles in the differentiated myotubes.

In parallel with highlighting the myogenic/anabolic transcriptional profile of the differentiated myotubes in response to different loading conditions, this study also



Figure 3. Effects of cyclic mechanical stretch on the expression of Atrophy Factors. Quantitative analysis of (A) Myostatin, (B) MuRF1 and $(C)$ Atrogin/MAFbx mRNA expression in myotubes subjected to mechanical stretch compared to control (non-stretched myotubes); The $m R N A$ values of atrophy factors in stretched myotubes were normalized to the corresponding GAPDH $m R N A$ and are expressed as fold changes compared to control. Percentages on the columns represent the degree of elongation of the cell culture surface. Note that in the selected stretching protocols as the elongation and frequency $(\mathrm{Hz})$ decrease the duration (Time) increases. * significantly different compared to control; ${ }^{a}$ significantly different compared to $15 \%$ elongation; ${ }^{\text {significantly }}$ different compared to $10 \%$ elongation $/ 1 \mathrm{~Hz}$; \#significantly different compared to $10 \%$ elongation $/ 0.25 \mathrm{~Hz} ;{ }^{¥}$ significantly different compared to $2 \%$ elongation $/ 12 \mathrm{~h}$; Mean + SE of 3 independent experiments performed in triplicate; $p<0.05$.

examined the expression responses of muscle atrophy genes to the various mechanical stimuli. Although the defined mechanisms regarding the role of those genes in driving an atrophic phenotype are yet to be fully elucidated, however, it is considered that myostatin has a role in actively inhibiting protein synthesis $(27,54)$, whilst MuRF1 and Atrogin-1 are thought to act through the ubiquitinproteasome pathway $(6,55,56)$. Specifically, myostatin is a negative regulator of myogenesis by deregulating MyoD activity (57). Similarly, Atrogin-1 appears to be negatively involved in myogenesis by polyubquitination and degradation of Myogenin (24), while MuRF1 plays a major role in regulating skeletal muscle mass by controlling a variety of muscle cell catabolic processes (35).

Our study revealed that, in contrast to the IGF-1 isoforms responses, the stretching protocol with the highest strain/frequency along with the shortest duration resulted in significant increases of myostatin and Atrogin-1. Inversely, the low strain/frequency for $12 \mathrm{~h}$ stretching was the only one that led to significant down-regulation of all three atrophy genes 
A

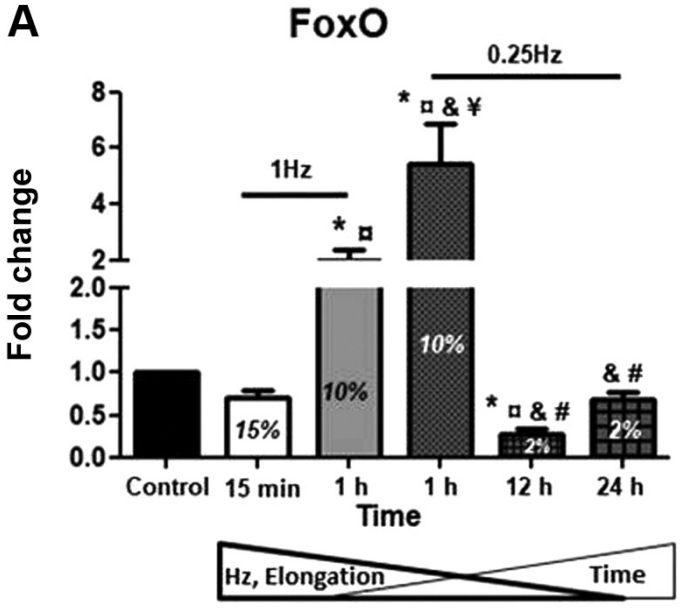

B

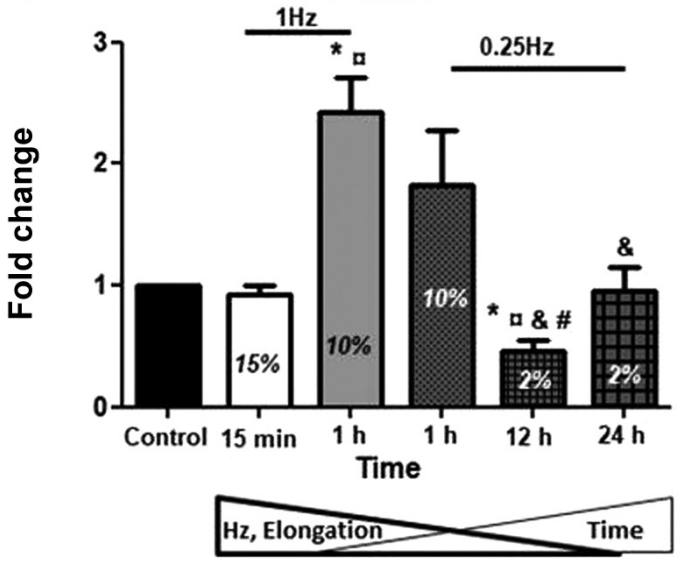



Figure 4. Effects of cyclic mechanical stretch on the expression of proapoptotic factors. Quantitative analysis of (A) FoxO, (B) FUCA and $(C)$ p53 mRNA expression in myotubes subjected to mechanical stretch compared to control (non-stretched myotubes); The mRNA values of apoptotic factors in stretched myotubes were normalized to the corresponding GAPDH mRNA and are expressed as fold changes compared to control. Percentages on the columns represent the degree of elongation of the cell culture surface. Note that in the selected stretching protocols as the elongation and frequency $(\mathrm{Hz})$ decrease the duration (Time) increases. *significantly different compared to control; ${ }^{a}$ significantly different compared to $15 \%$ elongation; \& significantly different compared to $10 \%$ elongation $/ 1 \mathrm{~Hz}$; ${ }^{\#}$ significantly different compared to $10 \%$ elongation $/ 0.25 \mathrm{~Hz}$; ${ }^{¥}$ significantly different compared to $2 \%$ elongation $/ 12 \mathrm{~h}$; Mean $+S E$ of 3 independent experiments performed in triplicate; $p<0.05$. examined (Figures 2 and 3). These findings suggest a multiple beneficial effect of the low strain/frequency of intermediate duration mechanical stretching, which simultaneously downregulates muscle atrophy program and up-regulates myogenic/growth factors in advanced differentiation myotubes.

Various pro-apoptotic factors may potentially be involved in myogenic differentiation; FoxO is a fate decider within the myogenic lineage as opposed to an inducer of the myogenic program (58), p53 suppresses muscle differentiation at the Myogenin step (32), while FUCA inhibits cell growth and induces cell death (59). In our model of applying mechanical stretching on differentiated myoblasts, the loading protocols using a $10 \%$ strain for $1 \mathrm{~h}$ resulted in the up-regulation of those factors regardless of the stretching frequency; nevertheless, frequency appeared to be the determinant factor for differentiating these increases (Figure 4). Inversely, low strain/low frequency of long duration stretching protocols decreased their expression similarly to the atrophy genes and in contrast to the myogenic factors' responses (Figures 1, 3 and 4). Our findings indicate that there might be a threshold (or range) of strain and/or duration of mechanical loading for the induction of detrimental effects on myotubes (60-62), such as the up-regulation of those apoptosis-related factors, since they were induced under specific strain and duration of loading.

Overall, our study revealed that a specific mechanical loading protocol (i.e., low strain/frequency of intermediate duration) was beneficial for the differentiated myotubes, not only by up-regulating myogenic and anabolic factors but also by down-regulating atrophy and pro-apoptotic genes.

\section{Conclusion}

Muscle cells are mechanosensitive responding in several ways to mechanical signals, produced from native environmental cues, in order to maintain their homeostasis and adapt to external loading. In vitro models of cell mechanical loading can greatly contribute to the improvement of our understanding of cellular mechanotransduction and molecular responses of skeletal muscle cells to loading. This study demonstrated that 
varying the components of mechanical stretching (i.e., elongation, frequency, duration) applied on myotubes in vitro, resulted in different, beneficial or detrimental, effects on their myogenic lineage, indicating specific features of loading for regulating the myogenic/anabolic program. These findings may be a valuable resource to be utilized as a database for developing more focused in vitro experimental designs to characterize the cellular and molecular mechanisms of mechanical loading-induced muscle adaptations.

\section{Conflicts of Interest}

The Authors declare no conflicts of interest in relation to this study.

\section{Authors' Contributions}

AM designed and performed the study, contributed the experimental process, analyzed data and wrote the manuscript; AP wrote and reviewed the manuscript, EZ contributed to the experimental process; OT , contributed to the experimental process; AC analyzed data and reviewed the manuscript; and MK had input in the experimental design, and reviewed the manuscript. The Authors have read and approved the final manuscript.

\section{References}

1 Passey S, Martin N, Player D and Lewis MP: Stretching skeletal muscle in vitro: Does it replicate in vivo physiology? Biotechnol Lett 33: 1513-1521, 2011. PMID: 21476095. DOI: 10.1007/s10529-011-0610-z

2 Tidball JG: Mechanical signal transduction in skeletal muscle growth and adaptation. J Appl Physiol (1985)98: 1900-1908, 2005. PMID: 15829723. DOI: 10.1152/japplphysiol.01178.2004

3 Soltow QA, Zeanah EH, Lira VA and Criswell DS: Cessation of cyclic stretch induces atrophy of c2c12 myotubes. Biochem Biophys Res Commun 434: 316-321, 2013. PMID: 23541574. DOI: 10.1016/j.bbrc.2013.03.048

4 Atherton PJ, Szewczyk NJ, Selby A, Rankin D, Hillier K, Smith $\mathrm{K}$, Rennie MJ and Loughna PT: Cyclic stretch reduces myofibrillar protein synthesis despite increases in fak and anabolic signalling in 16 cells. J Physiol 587: 3719-3727, 2009. PMID: 19470773. DOI: 10.1113/jphysiol.2009.169854

5 Stockdale FE and Miller JB: The cellular basis of myosin heavy chain isoform expression during development of avian skeletal muscles. Dev Biol 123: 1-9, 1987. PMID: 3305110. DOI: 10.1016/0012-1606(87)90420-9

6 Glass DJ: Signalling pathways that mediate skeletal muscle hypertrophy and atrophy. Nat Cell Biol 5: 87-90, 2003. PMID: 12563267. DOI: $10.1038 /$ ncb0203-87

7 Charge SB and Rudnicki MA: Cellular and molecular regulation of muscle regeneration. Physiol Rev 84: 209-238, 2004. PMID: 14715915. DOI: 10.1152 physrev.00019.2003

8 Karalaki M, Fili S, Philippou A and Koutsilieris M: Muscle regeneration: Cellular and molecular events. In Vivo 23: 779796, 2009. PMID: 19779115.

9 Kopantseva EE and Belyavsky AV: [key regulators of skeletal myogenesis]. Mol Biol (Mosk) 50: 195-222, 2016. PMID: 27239841. DOI: $10.7868 /$ S0026898416010079
10 Papasani MR, Wang G, Cheguru P, Julien DC, Hofwegen DV, Chapalamadugu KC, Brooking A and Read S: Interactions of muscle regulatory factors and isoforms of insulin growth factor1 in the muscle regenerative process. J Physiol 587: 2427-2428, 2009. PMID: 19483251. DOI: 10.1113/jphysiol.2009.169540

11 Philippou A, Halapas A, Maridaki M and Koutsilieris M: Type i insulin-like growth factor receptor signaling in skeletal muscle regeneration and hypertrophy. J Musculoskelet Neuronal Interact 7: 208-218, 2007. PMID: 17947802.

12 Barton ER, Park S, James JK, Makarewich CA, Philippou A, Eletto D, Lei H, Brisson B, Ostrovsky O, Li Z and Argon Y: Deletion of muscle grp94 impairs both muscle and body growth by inhibiting local igf production. FASEB J 26: 3691-3702, 2012. PMID: 22649033. DOI: 10.1096/fj.11-203026

13 Philippou A and Barton ER: Optimizing igf-i for skeletal muscle therapeutics. Growth Horm IGF Res 24: 157-163, 2014. PMID: 25002025. DOI: 10.1016/j.ghir.2014.06.003

14 Bikle DD, Tahimic C, Chang W, Wang Y, Philippou A and Barton ER: Role of IGF-i signaling in muscle bone interactions. Bone 80: 79-88, 2015. PMID: 26453498. DOI: 10.1016/j.bone.2015.04.036

15 Zou Y, Dong Y, Meng Q, Zhao Y and Li N: Incorporation of a skeletal muscle-specific enhancer in the regulatory region of igf1 upregulates igf1 expression and induces skeletal muscle hypertrophy. Sci Rep 8: 2781, 2018. PMID: 29426944. DOI: 10.1038/s41598-018-21122-5

16 Durzynska J, Philippou A, Brisson BK, Nguyen-McCarty M and Barton ER: The pro-forms of insulin-like growth factor i (IGFi) are predominant in skeletal muscle and alter IGF-i receptor activation. Endocrinology 154: 1215-1224, 2013. PMID: 23407451. DOI: $10.1210 /$ en.2012-1992

17 Papageorgiou E, Philippou A, Armakolas A, Christopoulos PF, Dimakakos A and Koutsilieris M: The human ec peptide: The active core of a progression growth factor with species-specific mode of action. Hormones (Athens) 15: 423-434, 2016. PMID: 27838607. DOI: 10.14310/horm.2002.1699

18 Philippou A, Papageorgiou E, Bogdanis G, Halapas A, Sourla A, Maridaki M, Pissimissis N and Koutsilieris M: Expression of igf-1 isoforms after exercise-induced muscle damage in humans: Characterization of the MGF e peptide actions in vitro. In Vivo 23: 567-575, 2009. PMID: 19567392.

19 Vassilakos G, Philippou A and Koutsilieris M: Identification of the igf-1 processing product human ec/rodent eb peptide in various tissues: Evidence for its differential regulation after exerciseinduced muscle damage in humans. Growth Horm IGF Res 32: 22-28, 2016. PMID: 27836414. DOI: 10.1016/j.ghir.2016.11.001

20 Tsao J, Vernet DA, Gelfand R, Kovanecz I, Nolazco G, Bruhn KW and Gonzalez-Cadavid NF: Myostatin genetic inactivation inhibits myogenesis by muscle-derived stem cells in vitro but not when implanted in the mdx mouse muscle. Stem Cell Res Ther 4: 4, 2013. PMID: 23295128. DOI: $10.1186 /$ scrt 152

21 Bodine SC, Latres E, Baumhueter S, Lai VK, Nunez L, Clarke BA, Poueymirou WT, Panaro FJ, Na E, Dharmarajan K, Pan ZQ, Valenzuela DM, DeChiara TM, Stitt TN, Yancopoulos GD and Glass DJ: Identification of ubiquitin ligases required for skeletal muscle atrophy. Science 294: 1704-1708, 2001. PMID: 11679633. DOI: $10.1126 /$ science. 1065874

22 Gomes MD, Lecker SH, Jagoe RT, Navon A and Goldberg AL: Atrogin-1, a muscle-specific f-box protein highly expressed during muscle atrophy. Proc Natl Acad Sci USA 98: 1444014445, 2001. PMID: 11717410. DOI: 10.1073/pnas.251541198 
23 Dapp C, Schmutz S, Hoppeler H and Fluck M: Transcriptional reprogramming and ultrastructure during atrophy and recovery of mouse soleus muscle. Physiol Genomics 20: 97-107, 2004. PMID: 15479860 . DOI: 10.1152 physiolgenomics.00100.2004

24 Jogo M, Shiraishi S and Tamura TA: Identification of mafbx as a myogenin-engaged f-box protein in scf ubiquitin ligase. FEBS Lett 583: 2715-2719, 2009. PMID: 19631210. DOI: $10.1016 /$ j.febslet.2009.07.033

25 Lokireddy S, Wijesoma IW, Sze SK, McFarlane C, Kambadur R and Sharma M: Identification of atrogin-1-targeted proteins during the myostatin-induced skeletal muscle wasting. Am J Physiol Cell Physiol 303: C512-529, 2012. PMID: 22673621. DOI: 10.1152/ajpcell.00402.2011

26 Schiaffino S, Dyar KA, Ciciliot S, Blaauw B and Sandri M: Mechanisms regulating skeletal muscle growth and atrophy. FEBS J 280: 4294-4314, 2013. PMID: 23517348. DOI: $10.1111 /$ febs. 12253

27 McPherron AC, Lawler AM and Lee SJ: Regulation of skeletal muscle mass in mice by a new tgf-beta superfamily member. Nature 387: 83-90, 1997. PMID: 9139826. DOI: 10.1038/387083a0

28 Morissette MR, Cook SA, Buranasombati C, Rosenberg MA and Rosenzweig A: Myostatin inhibits igf-i-induced myotube hypertrophy through akt. Am J Physiol Cell Physiol 297: C11241132, 2009. PMID: 19759331. DOI: 10.1152/ajpcell.00043.2009

29 Trendelenburg AU, Meyer A, Rohner D, Boyle J, Hatakeyama $\mathrm{S}$ and Glass DJ: Myostatin reduces akt/torc1/p70s6k signaling, inhibiting myoblast differentiation and myotube size. Am J Physiol Cell Physiol 296: C1258-1270, 2009. PMID: 19357233. DOI: 10.1152/ajpcell.00105.2009

30 Schwartz LM: Atrophy and programmed cell death of skeletal muscle. Cell Death Differ 15: 1163-1169, 2008. PMID: 18483492. DOI: $10.1038 /$ cdd 2008.68

$31 \mathrm{Wu}$ YJ, Fang YH, Chi HC, Chang LC, Chung SY, Huang WC, Wang $\mathrm{XW}$, Lee $\mathrm{KW}$ and Chen SL: Insulin and licl synergistically rescue myogenic differentiation of foxo1 overexpressed myoblasts. PLoS One 9: e88450, 2014. PMID: 24551104. DOI: 10.1371/journal.pone.0088450

32 Yang ZJ, Broz DK, Noderer WL, Ferreira JP, Overton KW, Spencer SL, Meyer T, Tapscott SJ, Attardi LD and Wang CL: P53 suppresses muscle differentiation at the myogenin step in response to genotoxic stress. Cell Death Differ 22: 560-573, 2015. PMID: 25501595. DOI: 10.1038/cdd.2014.189

33 Philippou A, Minozzo FC, Spinazzola JM, Smith LR, Lei H, Rassier DE and Barton ER: Masticatory muscles of mouse do not undergo atrophy in space. FASEB J 29: 2769-2779, 2015. PMID: 25795455. DOI: 10.1096/fj.14-267336

34 Hornberger TA, Mateja RD, Chin ER, Andrews JL and Esser KA: Aging does not alter the mechanosensitivity of the p38, p70s6k, and jnk2 signaling pathways in skeletal muscle. J Appl Physiol (1985)98: 1562-1566, 2005. PMID: 15361519. DOI: 10.1152/japplphysiol.00870.2004

35 Hornberger TA, Armstrong DD, Koh TJ, Burkholder TJ and Esser KA: Intracellular signaling specificity in response to uniaxial vs. Multiaxial stretch: Implications for mechanotransduction. Am J Physiol Cell Physiol 288: C185-194, 2005. PMID: 15371259. DOI: 10.1152/ajpcell.00207.2004

36 Hua W, Zhang M, Wang Y, Yu L, Zhao T, Qiu X and Wang L: Mechanical stretch regulates microrna expression profile via nfkappab activation in c2c12 myoblasts. Mol Med Rep 14: 50845092, 2016. PMID: 27840929. DOI: 10.3892/mmr.2016.5907
37 Moorwood C, Philippou A, Spinazzola J, Keyser B, Macarak EJ and Barton ER: Absence of gamma-sarcoglycan alters the response of p70s6 kinase to mechanical perturbation in murine skeletal muscle. Skelet Muscle 4: 13, 2014. PMID: 25024843. DOI: $10.1186 / 2044-5040-4-13$

38 Vining KH and Mooney DJ: Mechanical forces direct stem cell behaviour in development and regeneration. Nat Rev Mol Cell Biol 18: 728-742, 2015. PMID: 29115301. DOI: $10.1038 / \mathrm{nrm} .2017 .108$

39 Marshall KL and Lumpkin EA: The molecular basis of mechanosensory transduction. Adv Exp Med Biol 739: 142-155, 2012. PMID: 22399400. DOI: 10.1007/978-1-4614-1704-0_9

40 Vining KH and Mooney DJ: Mechanical forces direct stem cell behaviour in development and regeneration. Nature reviews. Mol Cell Biol 18: 728-742, 2017. PMID: 29115301. DOI: 10.1038/nrm.2017.108

41 Comai G, Sambasivan R, Gopalakrishnan S and Tajbakhsh S: Variations in the efficiency of lineage marking and ablation confound distinctions between myogenic cell populations. Dev Cell 31: 654-667, 2015. PMID: 25490270. DOI: 10.1016/j.devcel.2014.11.005

42 Zammit PS: Function of the myogenic regulatory factors myf5, myod, myogenin and mrf4 in skeletal muscle, satellite cells and regenerative myogenesis. Semin Cell Dev Biol 72: 19-32, 2017. PMID: 29127046. DOI: 10.1016/j.semcdb.2017.11.011

43 Yoshida N, Yoshida S, Koishi K, Masuda K and Nabeshima Y: Cell heterogeneity upon myogenic differentiation: Downregulation of myod and myf-5 generates 'reserve cells'. J Cell Sci 111(Pt 6): 769-779, 1998. PMID: 9472005.

44 Pavlath GK, Dominov JA, Kegley KM and Miller JB: Regeneration of transgenic skeletal muscles with altered timing of expression of the basic helix-loop-helix muscle regulatory factor mrf4. Am J Pathol 162: 1685-1691, 2003. PMID: 12707053. DOI: 10.1016/S0002-9440(10)64303-9

45 Florini JR, Ewton DZ and Roof SL: Insulin-like growth factor-i stimulates terminal myogenic differentiation by induction of myogenin gene expression. Mol Endocrinol 5: 718-724, 1991. PMID: 1649394. DOI: 10.1210/mend-5-5-718

46 Kook SH, Son YO, Choi KC, Lee HJ, Chung WT, Hwang IH and Lee JC: Cyclic mechanical stress suppresses myogenic differentiation of adult bovine satellite cells through activation of extracellular signal-regulated kinase. Mol Cell Biochem 309: 133141, 2008. PMID: 18008139. DOI: 10.1007/s11010-007-9651-y

47 Kumar A, Murphy R, Robinson P, Wei L and Boriek AM: Cyclic mechanical strain inhibits skeletal myogenesis through activation of focal adhesion kinase, rac-1 gtpase, and nf-kappab transcription factor. FASEB J 18: 1524-1535, 2004. PMID: 15466361. DOI: 10.1096/fj.04-2414com

48 Zhan M, Jin B, Chen SE, Reecy JM and Li YP: Tace release of tnf-alpha mediates mechanotransduction-induced activation of p38 mapk and myogenesis. J Cell Sci 120: 692-701, 2007. PMID: 17264149. DOI: $10.1242 /$ jcs.03372

49 Philippou A, Maridaki M, Halapas A and Koutsilieris M: The role of the insulin-like growth factor 1 (igf-1) in skeletal muscle physiology. In Vivo 21: 45-54, 2007. PMID: 17354613.

50 Goldspink G: Mechanical signals, igf-i gene splicing, and muscle adaptation. Physiology (Bethesda, Md.) 20: 232-238, 2005. PMID: 16024511. DOI: 10.1152/physiol.00004.2005

51 Juffer $\mathrm{P}$, Bakker $\mathrm{AD}$, Klein-Nulend $\mathrm{J}$ and Jaspers RT: Mechanical loading by fluid shear stress of myotube glycocalyx 
stimulates growth factor expression and nitric oxide production. Cell Biochem Biophys 69: 411-419, 2014. PMID: 24402674. DOI: $10.1007 / \mathrm{s} 12013-013-9812-4$

52 Rauch C and Loughna PT: Static stretch promotes mef2a nuclear translocation and expression of neonatal myosin heavy chain in c2c12 myocytes in a calcineurin- and p38-dependent manner. Am J Physiol Cell Physiol 288: C593-605, 2005. PMID: 15483225. DOI: 10.1152/ajpcell.00346.2004

53 Perrone CE, Fenwick-Smith D and Vandenburgh HH: Collagen and stretch modulate autocrine secretion of insulin-like growth factor-1 and insulin-like growth factor binding proteins from differentiated skeletal muscle cells. J Biol Chem 270: 20992106, 1995. PMID: 7530717. DOI: 10.1074/jbc.270.5.2099

54 Sharples AP and Stewart CE: Myoblast models of skeletal muscle hypertrophy and atrophy. Curr Opin Clin Nutr Metab Care 14: 230-236, 2011. PMID: 21460719. DOI: 10.1097/MCO.0b013e3283457ade

55 Gumucio JP and Mendias CL: Atrogin-1, murf-1, and sarcopenia. Endocrine 43: 12-21, 2013. PMID: 22815045. DOI: 10.1007/s12020-012-9751-7

56 Yang Y, Jemiolo B and Trappe S: Proteolytic mrna expression in response to acute resistance exercise in human single skeletal muscle fibers. J Appl Physiol 101: 1442-1450, 2006. PMID: 16840578. DOI: 10.1152/japplphysiol.00438.2006

57 Langley B, Thomas M, Bishop A, Sharma M, Gilmour S and Kambadur R: Myostatin inhibits myoblast differentiation by down-regulating myod expression. J Biol Chem 277: 4983149840, 2002. PMID: 12244043. DOI: 10.1074/jbc.M204291200

58 Kitamura T, Kitamura YI, Funahashi Y, Shawber CJ, Castrillon DH, Kollipara R, DePinho RA, Kitajewski J and Accili D: A foxo/notch pathway controls myogenic differentiation and fiber type specification. J Clin Invest 117: 2477-2485, 2007. PMID: 17717603. DOI: $10.1172 /$ JCI32054
59 Ezawa I, Sawai Y, Kawase T, Okabe A, Tsutsumi S, Ichikawa H, Kobayashi Y, Tashiro F, Namiki H, Kondo T, Semba K, Aburatani H, Taya Y, Nakagama H and Ohki R: Novel p53 target gene fucal encodes a fucosidase and regulates growth and survival of cancer cells. Cancer Sci 107: 734-745, 2016. PMID: 26998741. DOI: $10.1111 /$ cas.12933

60 Tsivitse SK, Mylona E, Peterson JM, Gunning WT and Pizza FX: Mechanical loading and injury induce human myotubes to release neutrophil chemoattractants. Am J Physiol Cell Physiol 288: C721-729, 2005. PMID: 15548571. DOI: 10.1152/ajpcell. 00237.2004

61 Nguyen HX, Lusis AJ and Tidball JG: Null mutation of myeloperoxidase in mice prevents mechanical activation of neutrophil lysis of muscle cell membranes in vitro and in vivo. J Physiol 565: 403-413, 2005. PMID: 15790660. DOI: 10.1113/jphysiol.2005.085506

62 Sampaolesi M, Yoshida T, Iwata Y, Hanada H and Shigekawa M: Stretch-induced cell damage in sarcoglycan-deficient myotubes. Pflugers Arch 442: 161-170, 2001. PMID: 11417209. DOI: $10.1007 / \mathrm{s} 004240100516$ 